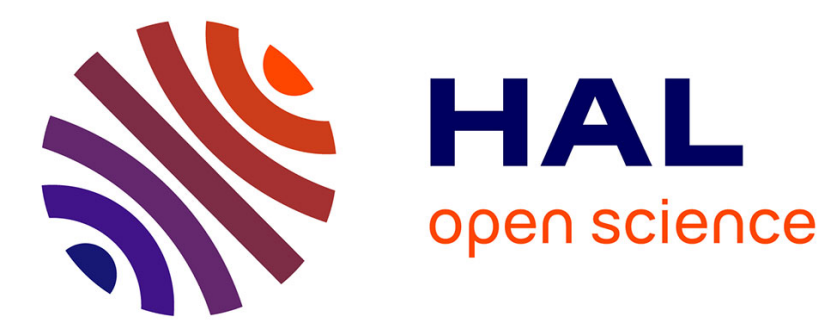

\title{
Probing heterogeneity of cortical bone with ultrasound axial transmission
}

Josquin Foiret, Quentin Grimal, Maryline Talmant, Roberto Longo, Pascal

Laugier

\section{- To cite this version:}

Josquin Foiret, Quentin Grimal, Maryline Talmant, Roberto Longo, Pascal Laugier. Probing heterogeneity of cortical bone with ultrasound axial transmission. IEEE Transactions on Ultrasonics, Ferroelectrics and Frequency Control, 2012, 10.1109/TUFFC.2013.2549 . hal-01586161

\section{HAL Id: hal-01586161 \\ https://hal.science/hal-01586161}

Submitted on 12 Feb 2018

HAL is a multi-disciplinary open access archive for the deposit and dissemination of scientific research documents, whether they are published or not. The documents may come from teaching and research institutions in France or abroad, or from public or private research centers.
L'archive ouverte pluridisciplinaire HAL, est destinée au dépôt et à la diffusion de documents scientifiques de niveau recherche, publiés ou non, émanant des établissements d'enseignement et de recherche français ou étrangers, des laboratoires publics ou privés. 


\title{
Probing Heterogeneity of Cortical Bone With Ultrasound Axial Transmission
}

\author{
Josquin Foiret, Quentin Grimal, Maryline Talmant, Roberto Longo, and Pascal Laugier, Member, IEEE
}

\begin{abstract}
In clinical examination of long cortical bones based on ultrasound axial transmission, the parameter currently used as indicator of bone fragility is the velocity of the first arriving signal (VFAS). VFAS is inherently related to the material properties of the bone site. However, experimental uncertainties may hide the true sensitivity of VFAS to elastic characteristics of bone. Measurements are performed with a multi-element compact array placed in contact with the bone. Therefore, VFAS measurements may be biased by variability imposed by geometrical irregularities of the sample below the probe and/or by probe misalignment. In this paper, we test the assumption that despite experimental errors, VFAS variations resulting from material properties can be measured. The methodology was to compare VFAS and velocities of compression bulk waves (VBWs) on carefully matched sites around the circumference of a test sample (bovine femur). VBW was mapped on bone cross-sections using a through-transmission technique. VBW and VFAS were highly correlated $\left[R^{2}=0.80\right.$, root mean square error $=23 \mathrm{~m} \cdot \mathrm{s}^{-1}, p<10^{-5}$ ] and the slope of the linear regression was close to 1 except in a part of the circumference with a pronounced curvature. In measurements performed with the same protocol as for clinical measurements, regions with different material properties (reflected by VBW) could be identified. This work demonstrates that within-specimen variations of material properties can be assessed with a technique available for in vivo measurements.
\end{abstract}

\section{INTRODUCTION}

$\mathrm{A}$ T ultrasound frequencies, long cortical bones such as the radius and tibia are assessed most commonly by the axial transmission (AT) technique. A linear arrangement of emitters and receivers along the bone axis allows excitation of several axially transmitted waves and capturing of the multi-component signal associated with them. Current clinical measurements rely mostly on the measurement of the velocity of the first arriving signal (VFAS). The VFAS was shown to be a relevant indicator of fracture risk [1], [2].

Cortical bone is known to be heterogeneous, i.e., its material properties vary around its circumference and along the bone axis [3]-[5]. This heterogeneity, which reflects bone remodeling and adaptation to loading conditions, may be relevant with regard to fracture risk prediction. Furthermore, measuring the heterogeneity could be a sig-

J. Foiret, Q. Grimal, M. Talmant, and P. Laugier are with the Laboratoire d'Imagerie Paramétrique, UMR 7623, Université Pierre et Marie Curie-Paris VI, Paris, France (e-mail: jfoiret@gmail.com).

R. Longo is with the Department of Mechanical Engineering, Vrije Universiteit Brussel, Brussels, Belgium. nificant advantage for advanced quantitative ultrasound (QUS) methods for the assessment of bone which attempt to recover bone properties from experimental signals based on a model following an inverse problem strategy. The reason for this is that models of cortical bone which account for random fluctuations of mechanical properties should allow a more accurate and more robust inversion [6], [7].

In the actual AT clinical protocol, VFAS is measured on several spatial positions for one individual. However the output of the protocol is a unique value of VFAS, which results from a sorting and a classification of several hundreds of raw VFAS values.

In this paper, we consider these raw data and test the assumption that, despite experimental uncertainties, their variations with spatial position reflect heterogeneity of cortical bone elastic properties. Our objective is to assess the potential of VFAS and of the established AT clinical protocol to measure the spatial variability of elastic properties in the cortical bone of an individual.

More precisely, the usual clinical protocols include an angular scan around the distal part of the forearm [8]-[10]. During one scan, several hundred VFAS values are recorded and the 95th percentile is retained as the indicator of bone quality (Fig. 1). Note that signals recorded when the contact is loose between the probe and the measured site or recorded during the movement of the probe (angular scan) are not taken into account. Usable raw data (points on Fig. 1) can be biased mostly by the residual experimental uncertainty, which includes misalignment of the probe with respect to bone axis and effects resulting from local irregular geometry. On standard geometry and homogeneous materials, it was previously shown that misalignment leads to a systematic decrease of VFAS and this motivated the choice of the highest VFAS value (95th percentile) to design a robust clinical parameter. Finally, for that parameter, a precision of $\pm 20 \mathrm{~m} \cdot \mathrm{s}^{-1}$ can be achieved in vivo (inter-operator reproducibility using the clinical protocol) [10]. This precision was close to the one obtained in vitro on human radius samples (with repeated measurement including repositioning of the probe without angular scan). Therefore, during a clinical angular scanning performed on a patient, the VFAS variations observed can be due to different factors in addition to variations of elastic properties. It follows that the contribution of bone heterogeneity to the signal fluctuations may not be measurable.

Two AT probes working at center frequencies of 1 and $2 \mathrm{MHz}$ were used to measure one bone specimen at several circumferential locations following the protocol developed for the in vivo measurements. The spatial distribution of the material bulk wave velocities (VBW) of the specimen 


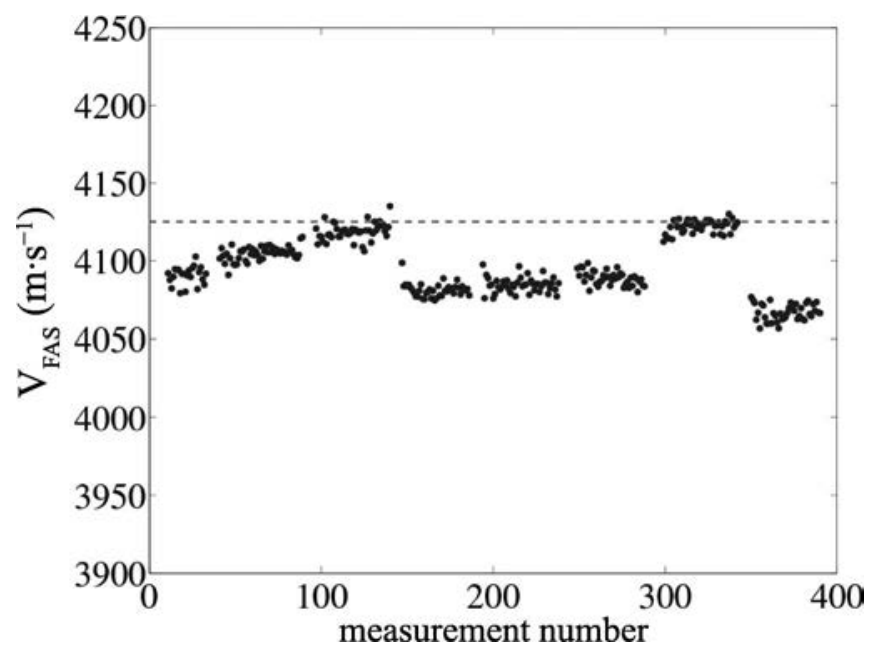

Fig. 1. Dots show a series of 400 experimental velocities of the first arriving signal (FAS) acquired in vivo on a human radius. As the probe is moved around the bone circumference, different values for the velocity VFAS are observable. The dashed horizontal line indicates the 95th percentile of the distribution, which is the retained value for VFAS.

was obtained subsequently and compared with the AT data to test the sensitivity of AT to bone heterogeneity.

Cortical thickness (C.Th) is a potential confounding factor affecting VFAS in addition to material properties. Because in this study we focused our attention on the sensitivity of VFAS to the spatial heterogeneity of elasticity, the experimental protocol has been specifically designed to avoid any influence of thickness on VFAS that may arise from local variations of bone C.Th. For this purpose, a bovine femur was used because of its large C.Th (more than $1 \mathrm{~cm}$ ). According to simulations of propagation on plates and tubular shells [11], VFAS measured with 1and $2-\mathrm{MHz}$ probes is independent of the thickness and is close to VBW, the velocity of compression bulk waves which propagate through the bone cross-section. Consequently, the first arriving signal (FAS) will not be affected by the finite thickness and will solely reflect the material properties, which are the focus of this work. With this approach, a direct comparison between VFAS and VBW can be achieved.

\section{Materials And Methods}

\section{A. Bone Specimen}

A bovine femur was obtained from a local butcher. Epiphyses were cut and soft tissues were removed from the diaphysis. The bone was stored at $-20^{\circ} \mathrm{C}$ between measurements. An orthonormal frame was defined, with $z$-axis aligned with the diaphysis axis (Fig. 2).

In the mid-diaphysis, a 1-cm-long region of interest was inspected with AT at several locations around the bone circumference. The length of the region of interest is approximately the length of the probe reception array along the bone axis.
Five roughly equal angular sectors, A, B, C, D, and E, were defined around the bone axis $z$. In addition, regularly spaced marks (5-mm spacing) were drawn around the circumference to allow a fine location of the probe. Sectors and 5-mm-spaced marks were used to co-localize bulk wave (BW) and AT measurements.

For BW measurement purposes, after AT measurements, three cross-sectional slabs of about 3-mm-thickness were cut with a precision saw (1-mm-thick blade; ISOMET 4000, Buehler SARL, Dardilly, France) under continuous irrigation in the part of the diaphysis measured with AT. Because of the strong ultrasound absorption in bone tissue at the frequencies used for BW measurements, it is hardly possible to measure a 1-cm-thick slab in through transmission. The 3 -mm-thickness is a compromise to optimize the signal-to-noise ratio of the BW measurements.

\section{B. AT Measurements}

AT measurements were taken with two custom-made bidirectional probes working at central frequencies of 1 and $2 \mathrm{MHz}$. The probes are made up of a linear arrangement of receivers located around the center and transmitters distributed on both sides of the receiving area. The bidirectional technique, consisting of calculating the harmonic mean of FAS velocity obtained in each opposite direction of propagation, was used for VFAS determination [12]. In each direction, the VFAS was measured as the ratio of inter-receiver distance over time delay using a linear regression. The first extremum of the FAS waveform was used to detect the FAS. AT measurements were performed by a trained operator using two distinct sequences:

1) The ordered sequence was used to compare AT measurement with site-matched BW velocities around the entire circumference. For this purpose, 25 regularly spaced locations were marked around the circumference (spacing between marks was $5 \mathrm{~mm}$ ). The

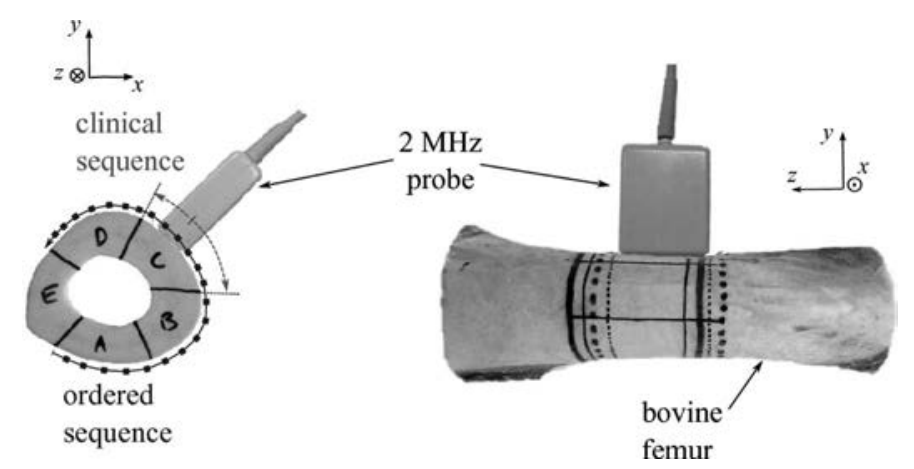

Fig. 2. (left) Cross-section of the bone and definition of the 4 angular sectors, A, B, C, D, which were measured with the clinical sequence. In this sequence, the probe is moved intermittently to cover the whole sector and record 3 cycles of 400 velocity of the first arriving signal (VFAS) measurements. In the ordered sequence, the probe is placed successively at known positions spaced by $5 \mathrm{~mm}$ and 100 VFAS values are recorded. (right) Diaphysis of the bovine femur with the $2-\mathrm{MHz}$ clinical probe aligned with the bone axis. 
probe was successively placed over each mark and 100 VFAS values were recorded at each location. The operator held the probe motionless over each mark during the measurement (no voluntary movement) and all 100 VFAS values recorded at one location were averaged.

2) The clinical sequence was adapted from the sequence of measurements used in clinical examination [8][10]. The probe was intermittently moved back and forth to cover each of the angular sectors, A, B, C, and D (Fig. 2). Three cycles of 400 VFAS measurements were performed in each sector. As in clinical measurements, the velocities biased by probe movements were removed. In addition, unstabilized velocities were eliminated according to the criterion used in clinical measurements: isolated unstable velocities were rejected by identifying VFAS variations larger than $\pm 50 \mathrm{~m} \cdot \mathrm{s}^{-1}$ inside a sliding window of $\pm 5 \mathrm{suc}-$ cessive measurements [10]. Note that in contrast to the actual in vivo clinical sequence, the data were not reduced to the 95 th percentile or the maximum value. As opposed to the ordered sequence, the clinical sequence did not record the position of the probe within the sector.

Preliminary measurements have shown that AT technique failed on sector $\mathrm{E}$ because of its pronounced surface curvature in the $x y$-plane. Accordingly, results are presented for sectors A through D only.

\section{BW Measurements}

The longitudinal VBW in the bone's axial direction ( $z$ axis) was measured at the points of a regular grid in the $x y$-plane of each slab. The grid step was $1 \mathrm{~mm}$, resulting in images of VBW with $1 \mathrm{~mm}^{2}$ pixels.

At each point, VBW was measured in through-transmission with a method similar to that described by Longo et al. [13]. The originality of the method, which evaluates the thickness simultaneously with the velocity, is that VBW is determined without a priori knowledge of the exact sample thickness at the measurement point. This is a critical point because the bone slab preparation method does not allow a precise control of its thickness.

The method was previously shown to recover the velocity with a reproducibility better than $0.05 \%$ on standard materials [13]. The emitting and receiving transducers (V312-SU, Panametrics, Waltham, MA; 6 mm diameter) and sample were immersed in a water tank. The emitting transducer was excited with a continuous compressed signal (function generator 33220A, Agilent Technologies Inc., Santa Clara, CA) resulting in an excitation signal with a $4 \mathrm{MHz}$ bandwidth centered at $5 \mathrm{MHz}$. The emitter and the receiver were placed on each side of the sample at 7 and $3 \mathrm{~cm}$, respectively. The sample was placed on a motorized support, allowing displacement along $x$ - and $y$-axes, controlled via a computer (MM 4006, Newport Corp., Irvine, CA). Each recorded signal is the result of the average of 16 temporal signals of $100 \mu$ s duration each. Signals were recorded by a digital oscilloscope (TDS 1012, Tektronix Inc., Beaverton, OR). A reference signal in water was acquired every 120 points. Water temperature was monitored and sound velocity in water was calculated from the formula of Lubbers and Graaf [14].

$\mathrm{BW}$ velocities were recovered from measurements of the transmission coefficient as a function of frequency, in the bandwidth 4 to $6 \mathrm{MHz}$, using an inversion scheme [13]. The differences between the experimental and theoretical transfer functions were minimized. The theoretical transfer function involves four parameters which are identified: through-sample time-of-flight, VBW, density, and attenuation. Because of the variability of VBW in cross-sections, several sets of initial values were systematically tested to ensure a correct convergence of the minimization algorithm. The behavior of the algorithm for the determination of VBW at each point on the edges of the samples was carefully checked; VBW values were rejected where necessary.

Validation of the method to map VBW in a cross-section with moderate thickness variability was achieved using a poly(methyl methacrylate) (PMMA) plate (see the Appendix).

Sectors A, B, C, and D were manually defined and divided in 25 sub-sectors corresponding to the marks (distant of $5 \mathrm{~mm}$ ) drawn on the circumference of the diaphysis (see Fig. 2). Finally, internal and external radial regions of interest were defined: a 3-mm-thick external (periosteal) region, referred to as the external ring, was obtained using a square structuring element of $3 \times 3$ pixels applied on the external edge; the internal region, referred to as the internal ring, consisted of the entire image with the external ring removed.

Imaging was performed separately on the three crosssectional slabs. For the analysis, the values of VBW in site-matched regions (sectors or sub-sectors) of the three slabs were pooled together.

\section{Data Analysis}

Data analysis consisted of investigating the variability of BW and AT measurements and elucidating their relationships. The hypothesis explored is that AT measurement variability can be largely explained by the BW heterogeneity.

Only VBW values in the external ring (Fig. 3) were processed because AT measurements probe this peripheral part of the cortical shell. The normality of VBW distributions in the different regions of interest was tested using Lilliefors test. The heterogeneity was analyzed by comparing the velocities in the external ring sectors (clinical sequence) and the velocities in the 5 -mm-spaced positions (ordered sequence). The statistical differences of VBW values in different regions of interest were tested using parametric or nonparametric tests (Kruskal-Wallis).

The possibility of measuring small variations of material properties with the AT probe was investigated by 


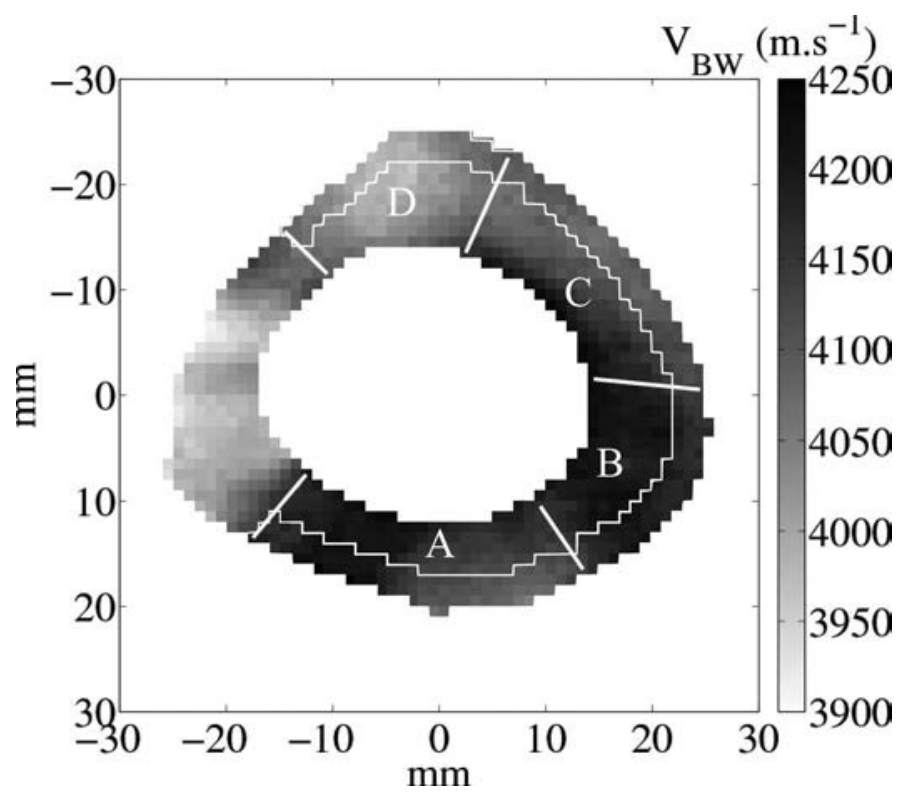

Fig. 3. A typical image of velocity of the bulk wave (VBW) values on a 3 -mm-thick slab. The velocity is shown in grayscale in meters per second. The pixel size is $1 \mathrm{~mm}^{2}$. The angular sectors A, B, C, and D and the external ring used in the data analysis are also represented.

comparing 25 site-matched VBW (each subsector) and VFAS (each measurement point of the ordered sequence) values. The trend of variations of the velocity values was evaluated with linear regression analysis.

The possibility of measuring bone heterogeneity with the clinical sequence was explored by comparing VFAS measured with the $1-$ and $2-\mathrm{MHz}$ probes and VBW in the external ring. In the clinical sequence measurement protocol, VFAS occurs in the form of several groups of similar values that fluctuate around a plateau value (Figs. 2 and $6)$. The average value and standard deviation of VFAS were calculated for each group separately.

Significance level for all tests was $5 \%$.
TABLE I. Velocity of the First Arriving Signal (VFAS) Values Obtained with the Clinical and Ordered Sequences.

\begin{tabular}{lcccc}
\hline & \multicolumn{2}{c}{ Clinical } & & Ordered \\
\cline { 2 - 3 } \cline { 5 - 5 } Frequency & $1 \mathrm{MHz}$ & $2 \mathrm{MHz}$ & & $2 \mathrm{MHz}$ \\
\hline Min(VFAS) $\left(\mathrm{m} \cdot \mathrm{s}^{-1}\right)$ & 3881 & 3890 & & 3916 \\
Max $($ VFAS $)\left(\mathrm{m} \cdot \mathrm{s}^{-1}\right)$ & 4215 & 4190 & & 4192 \\
Range $($ VFAS $)\left(\mathrm{m} \cdot \mathrm{s}^{-1}\right)$ & 334 & 300 & & 276 \\
\hline
\end{tabular}

\section{RESULTS}

The range of values obtained with the $2 \mathrm{MHz}$ probe with the ordered sequence and with the two probes for the clinical sequence are indicated in Table I.

\section{A. Maps of Bulk Wave Velocity in Cross-Sections}

The average VBW (all measurement points pooled) was $4130 \mathrm{~m} \cdot \mathrm{s}^{-1}$, in agreement with values found in literature [15]. Images of VBW reveal a complex pattern of the velocity distribution (Fig. 3). The distributions of VBW in each sector (A, B, C, or D) were significantly different from all of the other distributions. Concerning the radial variation, significant differences were observed between the external and internal ring, sector by sector $(\mathrm{A}, \mathrm{B}$, or C), except in sector D. In the subsectors of the external ring, VBW values were normally distributed, except in two subsectors. More than $70 \%$ of the 24 pairs of adjacent subsectors were significantly different. These differences appear mainly in sectors A and D and moderately in sectors B and C. Mean values and standard deviations (typically $18 \mathrm{~m} \cdot \mathrm{s}^{-1}$ ) of $\mathrm{VBW}$ in each subsector are plotted in Fig. 4. An asterisk indicates significant differences exist between the VBW distributions of 2 adjacent subsectors.

In other words, the statistical analysis suggests that subsectors on the external ring are areas in which VBW can be considered statistically homogeneous, i.e., VBW

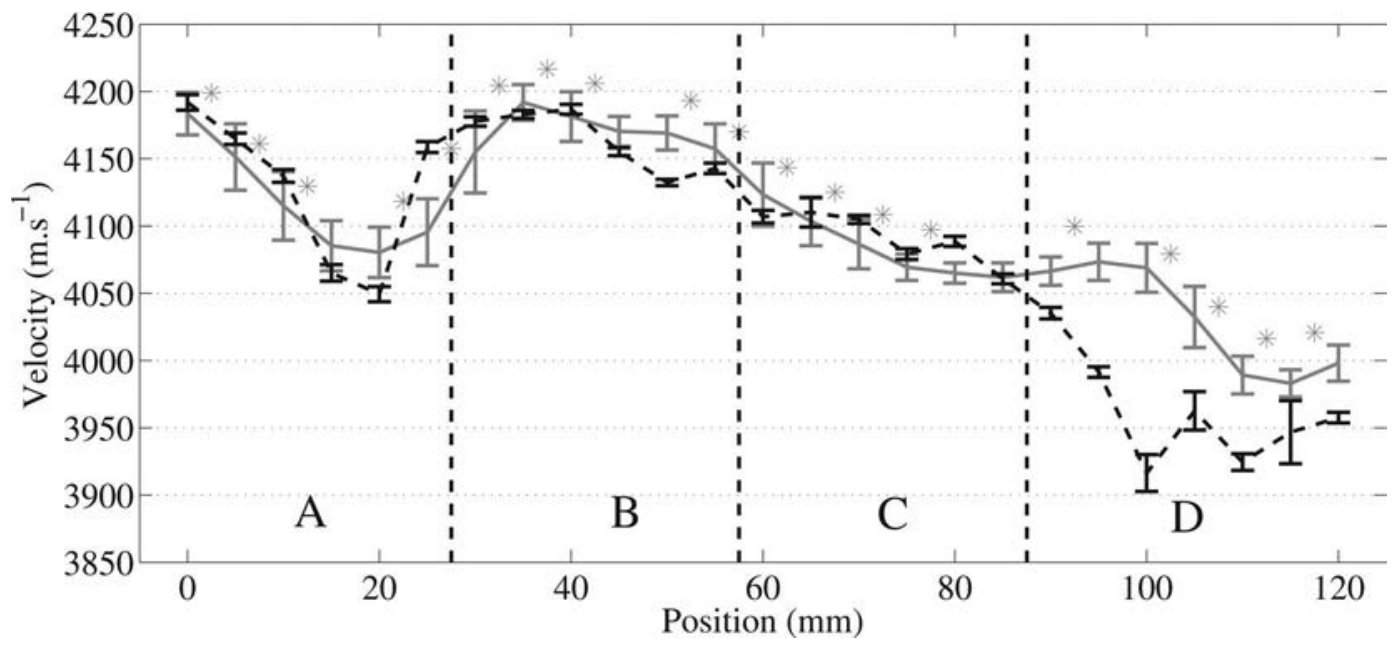

Fig. 4. Comparison between velocity of the first arriving signal (VFAS) obtained with the ordered axial transmission (AT) sequence at 2 MHz (dashed line) and the corresponding velocity of the bulk wave (VBW; continuous line) on the external ring. AT measurements were spaced out at 5 -mm intervals. Each point is the mean value of the distribution at the known circumferential position. The error bars indicate the standard deviation of the distribution. An asterisk indicates a significant difference between two adjacent distributions of VBW. 


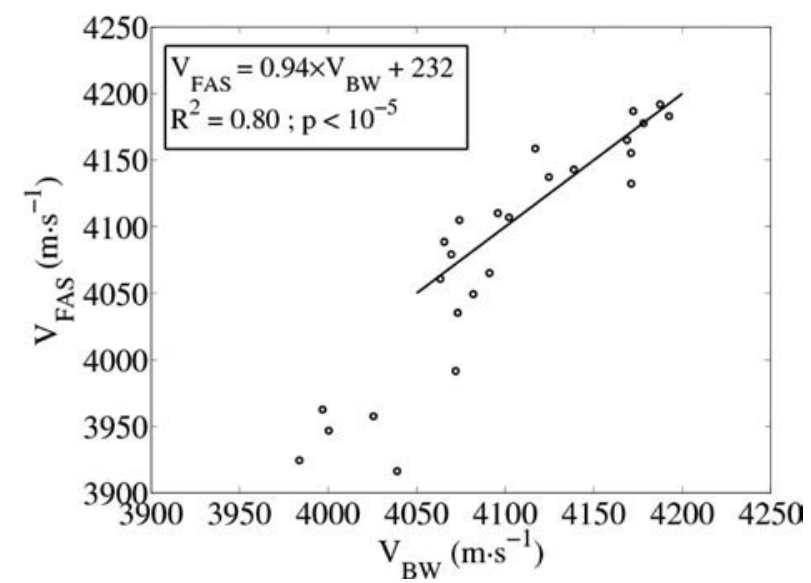

Fig. 5. Velocity of the first arriving signal (VFAS) acquired with the ordered sequence with the $2-\mathrm{MHz}$ axial transmission (AT) probe as a function of velocity of the bulk wave (VBW) in the subsectors. The linear regression is shown with the continuous line. Points belonging to the smallest values (sector D) were not considered for the regression.

are normally distributed. At larger scales (i.e., over several subsectors), VBW distributions are not normal, revealing significant differences between subsectors associated with the heterogeneity of VBW on bone.

\section{B. Ordered Sequence}

VFAS values measured with AT $(2 \mathrm{MHz})$ at successive marked positions closely reflect the local site-matched VBW in external ring sub-sectors, except mostly in sector $\mathrm{D}$ (Fig. 4). In sector $\mathrm{D}$, the concavity is more pronounced in the $y z$-plane. The average values of VBW in the sub-sectors on the external ring in sectors $\mathrm{A}, \mathrm{B}$, and $\mathrm{C}$ were significantly correlated to AT measurements in sitematched regions $\left(R^{2}=0.80, p<10^{-5}\right.$, root mean square error $=23 \mathrm{~m} \cdot \mathrm{s}^{-1}$; see Fig. 5). The slope of the linear regression was 0.94 (confidence interval: [0.71 to 1.18]).

\section{Clinical Sequence}

VFAS values obtained during the clinical sequence appear in groups around plateau values (Fig. 6), similar to in vivo measurements with the same protocol [10]. Each plateau is obtained when the operator does not move.

In each sector, VFAS plateau values and VBW follow the same trend (Fig. 7). Our way to relate VBW measurements to VFAS was to sort, in each sector separately, both VBW and plateau values in ascending order. In Fig. 7 , data are represented such that in each sector, values are spanned on the same arbitrary interval. The indicated VBW values are the average values in each external ring subsector.

\section{Discussion}

This work demonstrates that the in vivo sequence of measurements, applied in vitro to a bovine femur, allows
VFAS measurements sensitive to tissue heterogeneity. The clinical protocol set for human long bones includes 3 cycles of 400 VFAS measurements and intermittent back and forth circumferential motion. The protocol yields about 10 plateau values in each angular sector, which span the range of values that are expected in view of the material heterogeneity. The clinical protocol is thus found to be sufficient to probe the circumferential heterogeneity.

We have quantified the heterogeneity of material properties in terms of axial compression bulk wave velocity. These were measured with a particularly precise method which was developed previously [13]. The ordered sequence with the AT probe allowed a site-matched comparison of VBW and VFAS. In the linear model, up to $80 \%$ of VFAS variations were explained by the underlying heterogeneity of the material properties (VBW) in the external ring. We suggest that the unexplained VFAS variations are due to imperfect site-matching, misalignment of the AT probe with the bone axis, and precision of the device.

To consider the implication of the work for in vivo assessment of human bone, several points must be discussed.

1) First, we chose to measure a bovine femur, which has a high thickness-to-wavelength ratio; in that case, FAS was shown to be insensitive to thickness according to model predictions [11]. FAS corresponds to a lateral wave which is the trace on the surface of the compression bulk wave propagating in cortical bone. However, when cortical thickness is below or comparable to the wavelength, guided waves are involved in FAS, and VFAS is sensitive to thickness. For instance in the human forearm (radius), where cortical thickness is typically in the range 1 to $4 \mathrm{~mm}$ [16], [17], the variability of cortical thickness under the probe, contributing to the variability of VFAS in addition to that of material properties, must be taken into account.

2) Heterogeneity of material properties in bovine bone and human long bone may be different. To our

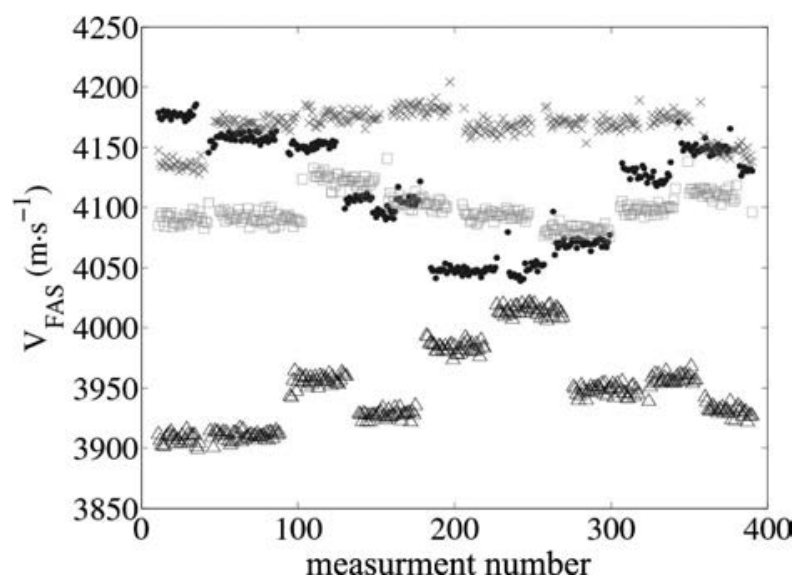

Fig. 6. Typical acquisition with the 2-MHz probe (clinical sequence). Velocity of the first arriving signal (VFAS) values were obtained after 400 measurements on the angular sectors $\mathrm{A}(\bullet), \mathrm{B}(\times), \mathrm{C}(\square)$, and $\mathrm{D}(\Delta)$. 


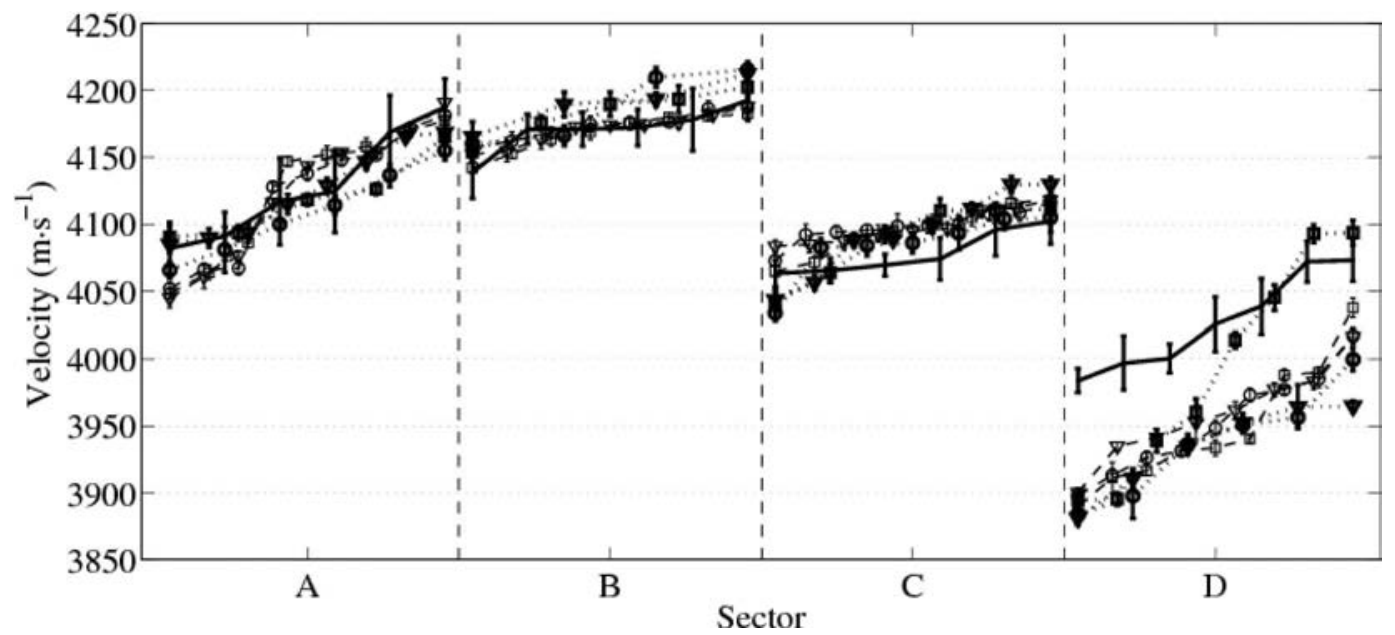

Fig. 7. Comparison between velocity of the bulk wave (VBW) data (continuous line) and the 3 cycles of velocity of the first arriving signal (VFAS) obtained with the clinical sequence at $1 \mathrm{MHz}$ (dotted line) and $2 \mathrm{MHz}$ (dashed line) for each sector, A to D. For the VBW data, each point is the mean value obtained in a subsector on the external ring. For the VFAS data, each point is the mean value obtained on a plateau as shown in Fig. 6 . The error bars indicate the standard deviation of the group of data around each plateau (VFAS) or in each subsector (VBW).

knowledge, the material heterogeneity of the human radius has not been documented at a length scale relevant to the present work (i.e., a few millimeters). Nevertheless, heterogeneity within cross-sections of human femurs has been observed [3]-[5].

3) This work shows that in most sites around the diaphysis circumference, on bovine bone, the surface is suited to a correct measurement. The geometry (surface shape) of the human radius and bovine femur diaphysis differs. The radius of curvature in the human bone is smaller, which may limit the precision of the measurements. The external shape of the bone is a limitation on the measurement of heterogeneity. The characteristic plateaus can be associated without ambiguity to a material property (VBW) only in those cases where the bone surface is regular enough.

4) In this work, we did not consider soft tissues between the probe and the bone. In a previous work, it was found that the thickness of soft tissues modeled by a water layer have a small effect on VFAS measurement [12]. However, heterogeneities of elastic properties in soft tissues, if any, may alter the sensitivity of our technique to bone heterogeneity. Note that with the bidirectional axial transmission technique, effects of uneven soft tissue thickness are automatically corrected [12].

The possibility of measuring heterogeneous material properties of cortical bone with a clinical device is likely to foster research on novel QUS indicators of fracture risks accounting for heterogeneity and novel inversion strategies to recover bone properties.

\section{Conclusion}

In this study, a direct comparison between FAS velocities (VFAS) in the axial transmission configuration and compression BW velocities (VBW) was made on a bovine femur.

VFAS was measured using a dedicated clinical device following two protocols: first, VFAS was measured at fixed known positions (ordered sequence) and second, clinical examination of angular sectors was performed (clinical sequence). A through-transmission technique was used to draw VBW maps of bone cross-sections. VBW and VFAS showed a good correlation for site-matched measurements. Concerning the clinical sequences, VFAS was shown to reflect the same fluctuations as VBW in angular sectors. However, these results with VFAS were found when the contact between the axial transmission probe and the bone surface was optimal. This work shows that VFAS closely reflects bone material heterogeneities when FAS is not affected by the thickness.

\section{Appendix}

In our experiment on the bovine femur, cutting slabs with a constant thickness showing less than $100-\mu \mathrm{m}$ variations proved difficult, because of the large cortical thickness and bone diameter. To assess the precision of velocity and thickness measurements with BW, a PMMA plate of varying thickness was prepared. Its average thickness was $4.65 \mathrm{~mm}$ with an almost linear variation of $20 \mu \mathrm{m} \cdot \mathrm{cm}^{-1}$. A part of the plate was sanded to increase the thickness variability. As shown on Fig. 8, determined thickness and velocity were found to be unrelated. The average BW velocity was $2757.2 \mathrm{~m} \cdot \mathrm{s}^{-1}$ with a standard deviation of $1.4 \mathrm{~m} \cdot \mathrm{s}^{-1}$ and no coherent fluctuations that would reveal any coupling with the thickness determination were visible. Estimated thicknesses were very close to those measured with a caliper, with differences less than $15 \mu \mathrm{m}$. Therefore, velocity measurements on bone were considered to be independent of the sample thickness. 


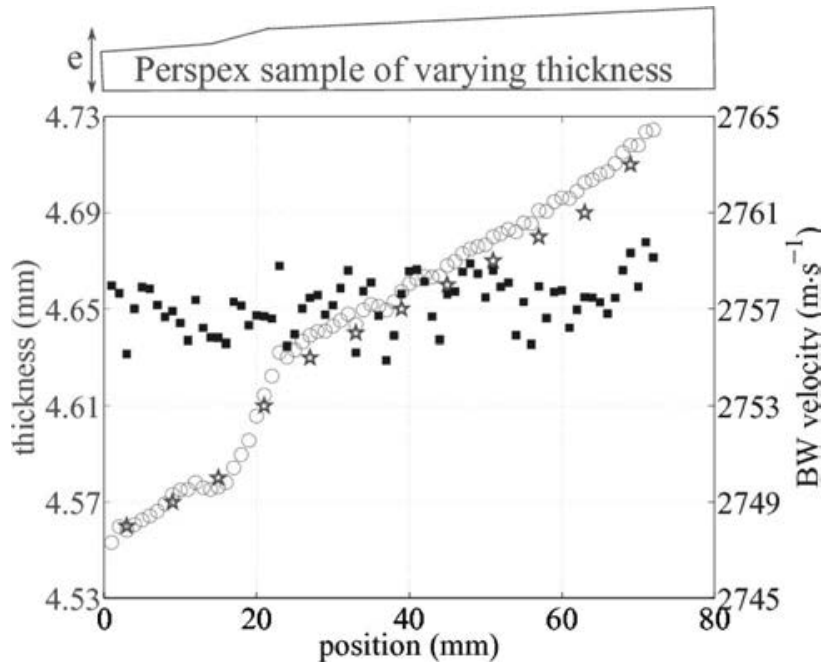

Fig. 8. Comparison between caliper-measured thicknesses (asterisks, precision $\pm 0.01 \mathrm{~mm}$ ) and estimated thicknesses (circles). Thickness is indicated on the left axis. Estimated velocities are shown by square markers. Velocity is indicated on the right axis. Note that the relative variation of velocity is less than $6 \mathrm{~m} \cdot \mathrm{s}^{-1}$.

\section{REFERENCES}

[1] R. Barkmann, E. Kantorovich, C. Singal, D. Hans, H. K. Genant, M. Heller, and C. C. Gluer, "A new method for quantitative ultrasound measurements at multiple skeletal sites-First results of precision and fracture discrimination," J. Clin. Densitom., vol. 3, no. 1 , pp. $1-7,2000$.

[2] C. F. Njeh, N. Shaw, J. M. Gardner-Medwin, C. M. Boivin, and T. R. Southwood, "Use of quantitative ultrasound to assess bone status in children with juvenile idiopathic arthritis," J. Clin. Densitom., vol. 3, no. 3, pp. 251-260, 2000.

[3] S. Bensamoun, J.-M. Gherbezza, J.-F. de Belleval, and M.-C. Ho Ba Tho, "Transmission scanning acoustic imaging of human cortical bone and relation with the microstructure," Clin. Biomech., vol. 19, no. 6, pp. 639-647, 2004.

[4] Q. Grimal, S. Haupert, D. Mitton, L. Vastel, and P. Laugier, "Assessment of cortical bone elasticity and strength: Mechanical testing and ultrasound provide complementary data," Med. Eng. Phys., vol. 31, no. 9, pp. 1140-1147, 2009.

[5] J. Grondin, Q. Grimal, K. Yamamoto, M. Matsukawa, A. Saïed, and P. Laugier, "Relative contributions of porosity and mineralized matrix properties to the bulk axial ultrasonic wave velocity in human cortical bone," Ultrasonics, vol. 52, pp. 467-471, 2012.

[6] K. Macocco, Q. Grimal, S. Naili, and C. Soize, "Elastoacoustic model with uncertain mechanical properties for ultrasonic wave velocity prediction: Application to cortical bone evaluation," J. Acoust. Soc. Am., vol. 119, no. 2, pp. 729-740, 2006.

[7] C. Desceliers, C. Soize, Q. Grimal, M. Talmant, and S. Naili, "Determination of the random anisotropic elasticity layer using transient wave propagation in a fluid-solid multilayer: Model and experiments," J. Acoust. Soc. Am., vol. 125, no. 4, pp. 2027-2034, 2009.

[8] E. Bossy, "Evaluation ultrasonore de l'os cortical par transmission axiale: Modelisation et expérimentation in vitro et in vivo," Ph.D. thesis, Laboratoire d'Imagerie Paramétrique, Université Paris 6, Paris, France, 2004, [Online]. Available: http://tel.archives -ouvertes.fr/tel-00003220

[9] M. Weiss, A. Ben-Shlomo, P. Hagag, and S. Ish-Shalom, "Discrimination of proximal hip fracture by quantitative ultrasound measurement at the radius," Osteoporos. Int., vol. 11, no. 5, pp. 411-416, 2000 .

[10] M. Talmant, S. Kolta, Ch. Roux, D. Haguenauer, I. Vedel, B. Cassou, E. Bossy, and P. Laugier, "In vivo performance evaluation of bi-directional ultrasonic axial transmission for cortical bone assessment," Ultrasound Med. Biol., vol. 35, no. 6, pp. 912-919, 2009.

[11] E. Bossy, M. Talmant, and P. Laugier, "Three-dimensional simulations of ultrasonic axial transmission velocity measurement on cor- tical bone models," J. Acoust. Soc. Am., vol. 115, no. 5, pt. 1, pp. 2314-2324, 2004.

[12] E. Bossy, M. Talmant, M. Defontaine, F. Patat, and P. Laugier, "Bidirectional axial transmission can improve accuracy and precision of ultrasonic velocity measurement in cortical bone: A validation on test materials," IEEE Trans. Ultrason. Ferroelectr. Freq. Control, vol. 51, no. 1, pp. 71-79, 2004.

[13] R. Longo, Q. Grimal, P. Laugier, S. Vanlanduit, and G. Patrick, "Simultaneous determination of acoustic velocity and density of a cortical bone slab: Ultrasonic model-based approach," IEEE Trans. Ultrason. Ferroelectr. Freq. Control, vol. 57, no. 2, pp. 496-500, 2010.

[14] J. Lubbers and R. Graaf, "A simple and accurate formula for the sound velocity in water," Ultrasound Med. Biol., vol. 24, no. 7, pp. 1065-1068, 1998

[15] S. Lees and D. Z. Klopholz, "Sonic velocity and attenuation in wet compact cow femur for the frequency range 5 to $100 \mathrm{MHz}$," Ultrasound Med. Biol., vol. 18, no. 3, pp. 303-308, 1992.

[16] E. Bossy, M. Talmant, F. Peyrin, L. Akrout, P. Cloetens, and P. Laugier, "An in vitro study of the ultrasonic axial transmission technique at the radius: 1-Mhz velocity measurements are sensitive to both mineralization and intracortical porosity," J. Bone Miner. Res., vol. 19, no. 9, pp. 1548-1556, 2004.

[17] H. Sievänen, S. Cheng, S. Ollikainen, and K. Uusi-Rasi, "Ultrasound velocity and cortical bone characteristics in vivo," Osteoporos. Int., vol. 12, no. 5, pp. 399-405, 2001. 